\title{
Response to Comment on "Replacing the NCCN's Blocks with Wheels: How Should Consideration of Societal Spending be Incorporated into Oncology Practice?"
}

\author{
Audrey $\operatorname{Tran}^{1} \cdot$ Vinay Prasad ${ }^{1,2,3}$ \\ Published online: 26 June 2020 \\ (c) Springer Nature Switzerland AG 2020
}

We appreciate the letter by Koh and Carlson [1] in response to our article Replacing the NCCN's Blocks with Wheels: How Should Consideration of Societal Spending be Incorporated into Oncology Practice? [2].

In our article [2], we noted that, by including information on total costs-i.e., costs to society-the National Comprehensive Cancer Network (NCCN) is tacitly encouraging providers and patients to consider this information when making treatment decisions. We believe the reply from Koh and Carlson [1] implies confusion within the NCCN about what they are doing.

There are two types of costs: costs to society and costs to the patient. The NCCN's blocks do not capture or report costs to the patient, which vary wildly according to specific insurance programs, out-of-pocket requirements, and year-to-date deductible payments. The NCCN has no way to know or provide this information. Instead, the NCCN is providing a crude estimate of societal spending. As the authors noted, "an estimate of overall relative total cost of a therapy, including but not limited to acquisition, administration, site of care, infusions, laboratory monitoring, antiemetics and growth factors, and toxicity management." There is no debate: this is a measure of societal and not personal costs.

This reply refers to the article available at https://doi.org/10.1007/ s40273-020-00938-y.

Vinay Prasad

vinayak.prasad@ucsf.edu

1 Oregon Health and Science University School of Medicine, Portland, USA

2 Department of Epidemiology and Biostatistics, UCSF, San Francisco, USA

3 Department of Epidemiology and Biostatistics, University of California, San Francisco, UCSF Mission Bay Campus, Mission Hall: Global Health \& Clinical Sciences Building, 550 16th St, 2nd Fl, San Francisco, CA 94158, USA
Theirs is only a rough estimate because it does not include downstream costs, such as hospitalizations or other therapies, which may be increased or decreased by certain regimens; it is also not a formal cost-effectiveness analysis. The NCCN provides this information-a crude measure of societal spending-to physicians. Assertions that "no precise cost is provided" do not alter the fact that relative societal costs by regimen are given.

There is no question that doctors will, to some degree, factor in societal costs when making treatment decisions. Drs. Koh and Carlson [1] deny that possibility, but it is inescapable. Our article discussed the implications of this [2].

If the authors instead wished to empower patients to make decisions that align with their own values and preferences, as they state, they would need to report patient-level costs, such as aggregate copays, and the need for subsequent therapy and hospitalization, which may also incur personal expenditure.

In short, we believe the letter by Koh and Carlson [1] shows that the NCCN misunderstands what is accomplished by the NCCN's evidence blocks. We encourage the NCCN to seek further consultation with experts in drug pricing and decision making.

\section{Compliance with Ethical Standards}

Conflict of interest Vinay Prasad has received research funding from Arnold Ventures; royalties from Johns Hopkins Press and Medscape; honoraria for conducting grand rounds and delivering lectures at universities, medical centers, non-profits, and professional societies; consulting fees from UnitedHealthcare; and speaking fees from Evicore. In addition, the Plenary Session podcast has Patreon backers. Audrey Tran has no conflict of interest. 


\section{References}

1. Koh W-J, Carlson RW. Comment on "Replacing the NCCN's Blocks with Wheels: How should consideration of societal spending be incorporated into oncology practice?" PharmacoEconomics. 2020. https://doi.org/10.1007/s40273-020-00938-y.

2. Tran AA, Prasad V. Replacing the NCCN's Blocks with Wheels: How should consideration of societal spending be incorporated into oncology practice? PharmacoEnconomics. 2020. https://doi. org/10.1007/s40273-020-00920-8. 